\title{
Infrared [Fe II] and Dust Emissions from Supernova Remnants
}

\author{
Bon-Chul Koo \\ Department of Physics and Astronomy, Seoul National University, \\ Seoul 151-747, KOREA \\ email: koo@astro.snu.ac.kr
}

\begin{abstract}
Supernova remnants (SNRs) are strong thermal emitters of infrared radiation. The most prominent lines in the near-infrared spectra of SNRs are [Fe II] lines. The [Fe II] lines are from shocked dense atomic gases, so they trace SNRs in dense environments. After briefly reviewing the physics of the [Fe II] emission in SNR shocks, I describe the observational results which show that there are two groups of SNRs bright in [Fe II] emission: middle-aged SNRs interacting with molecular clouds and young core-collapse SNRs in dense circumstellar medium. The SNRs belonging to the former group are also bright in near-infrared $\mathrm{H}_{2}$ emission, indicating that both atomic and molecular shocks are pervasive in these SNRs. The SNRs belonging to the latter group have relatively small radii in general, implying that most of them are likely the remnants of SN IIL/b or SN IIn that had strong mass loss before the explosion. I also comment on the "[Fe II]- $\mathrm{H}_{2}$ reversal" in SNRs and on using the [Fe II]-line luminosity as an indicator of the supernova $(\mathrm{SN})$ rate in galaxies. In the mid- and far-infrared regimes, thermal dust emission is dominant. The dust in SNRs can be heated either by collisions with gas species in a hot plasma or by radiation from a shock front. I discuss the characteristics of the infrared morphology of the SNRs interacting with molecular clouds and their dust heating processes. Finally, I give a brief summary of the detection of SN dust and crystalline silicate dust in SNRs.
\end{abstract}

Keywords. shock waves, ISM: supernova remnant, infrared: ISM

\section{Introduction}

Infrared (IR) covers 3 decade logarithmic scales in wavelength, from 1 to $1000 \mu \mathrm{m}$. This is the waveband in which we observe emission from dust, forbidden fine-structure lines from various metallic atoms and ions, molecular lines, and H-recombination lines. These diverse and unique emission features, together with their relatively small extinctions, make the IR band particularly useful for studying various physical and astrophysical processes related to shocks and supernova remnants (SNRs).

During the past 10 years, significant progress has been made in the IR study of SNRs as a result of space missions equipped with mid- and far-IR instruments and the development of wide-field IR cameras and broadband spectrometers. In this paper, I shall talk about two particular spectral features often found in SNRs: (1) [Fe II] emission lines in the near-IR (NIR) band, which is the most prominent NIR spectral feature in SNRs, and (2) dust continuum emission in mid- and far-IR spectra. For the [Fe II] lines, I briefly review the basic physics, summarize observational results, and then discuss the characteristics of [Fe II]-bright SNRs along with some related issues. For the dust emission, as there are other papers on this topic in this volume, I simply present some recent topics that are relevant to supernovae (SNe) and SNR environments. 

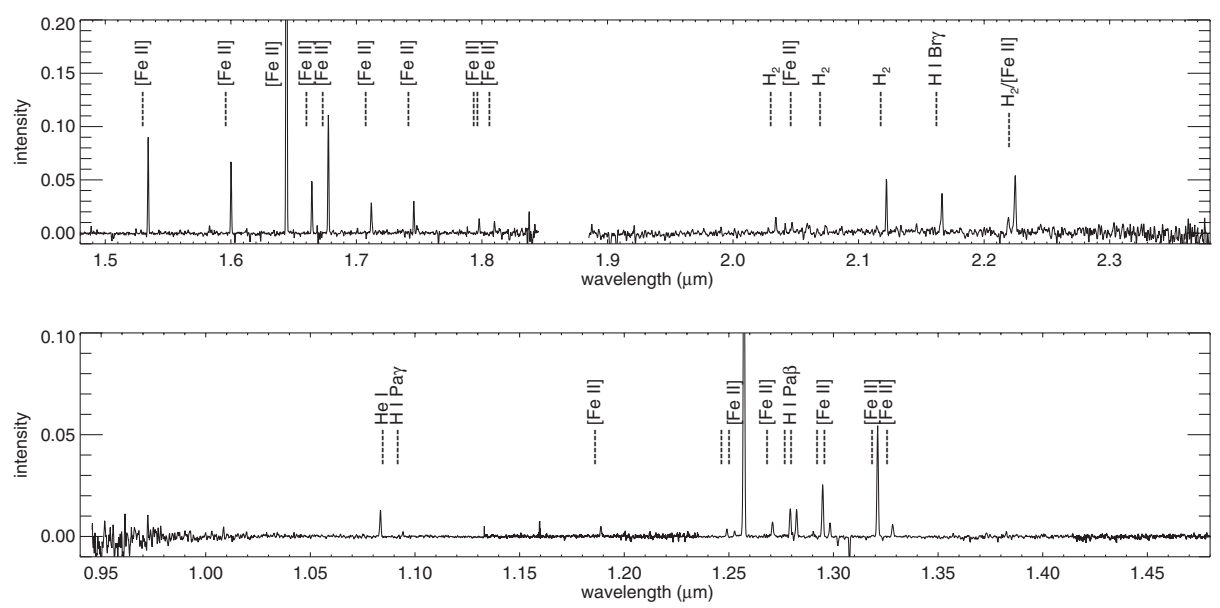

Figure 1. TripleSpec spectrum of the SNR G11.2-0.3, showing numerous [Fe II] lines and other emission lines (Courtesy of D.-S. Moon). The extinction to the source is large $\left(A_{V}=16 \mathrm{mag}\right)$, so that the observed line intensities can be significantly different from the intrinsic ones.

\section{NIR [Fe II] Emission from SNRs}

\subsection{NIR [Fe II] Emission Lines and J Shocks}

In the NIR spectra of SNRs, [Fe II] emission lines are usually the most prominent unless there is heavy-element-enriched SN ejecta (Fig. 1). This contrasts with photoionized HII regions where $\mathrm{H}$ recombination lines are much stronger; i.e., $[\mathrm{Fe} \mathrm{II}] 1.257 \mu \mathrm{m} / \mathrm{Pa} \beta(\sim 3 \times$ [Fe II] $1.644 \mu \mathrm{m} / \mathrm{Pa} \alpha)=2-8$ in SNRs whereas it is 0.013 in Orion (Oliva et al. 1989; Mouri et al. 2000). Such a large difference arises because Fe atoms in photoionized gas are in higher ionization stages and also probably because the Fe abundance in shocked gas is enhanced by dust destruction. Therefore, [Fe II] emission can be used as a tracer of fast radiative atomic shocks, although strong [Fe II] lines may be observable in sources ionized by X-rays, e.g., in active galactic nuclei (Mouri et al. 2000).

The $\mathrm{Fe}^{+}$ion has four ground terms, each of which has 3-5 closely-spaced levels to form a 16 level system (Pradhan \& Nahar 2011). The energy gap between the ground level and the excited levels is less than $1.3 \times 10^{4} \mathrm{~K}$, and thus, these levels are easily excited in the postshock cooling region. The emission lines resulting from the transitions among these levels appear in the visible to far-infrared bands (Fig. 2 right). In the NIR JHK bands, 10-20 [Fe II] lines are visible; these include the two strongest lines at 1.257 and $1.644 \mu \mathrm{m}$. The ratios of these lines provide a very good density diagnostic and an accurate measure of extinction to the emitting region.

[Fe II] emission lines in SNRs are mostly emitted from cooling gas behind radiative atomic shocks. Figure 2 (top left) shows the temperature profile in the postshock cooling layer of a radiative shock. At $N_{\mathrm{H}} \sim 5 \times 10^{17} \mathrm{~cm}^{-2}$ the cooling becomes important and the temperature abruptly drops to $\sim 8,000 \mathrm{~K}$. Then the temperature remains constant over an extended region, where the heating is maintained by UV radiation generated from the hot gas immediately behind the shock front. The corresponding profiles of $\mathrm{H}$ nuclei and electron densities are shown in the bottom left frame of Figure 2, together with the $\mathrm{Fe}^{+}$fraction profile. Note that, since the ionization potential of the iron atom is $7.90 \mathrm{eV}$, far-UV photons from the hot shocked gas can penetrate far downstream to maintain the ionization state of $\mathrm{Fe}^{+}$where $\mathrm{H}$ atoms are primarily neutral. Most of the [Fe II] emission, however, originates from the temperature plateau region where the ionization fraction is not too low, as shown in Figure 2. Numerical shock models covering some parameter 

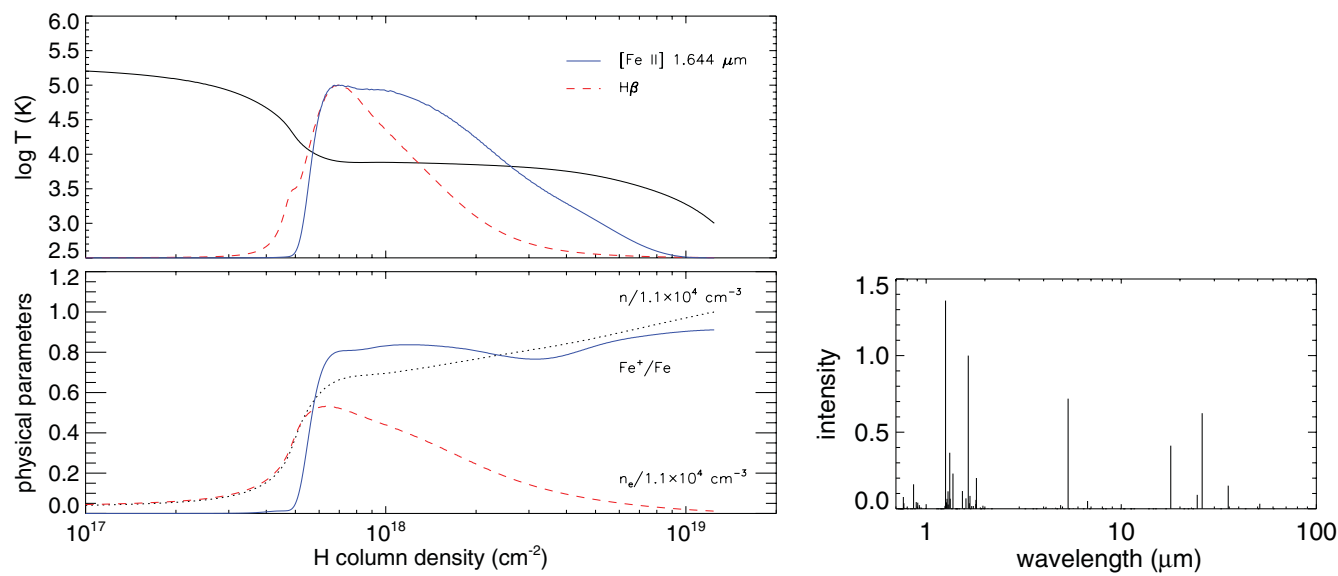

Figure 2. (Top left) Temperature profile as a function of swept-up H-nuclei column density for a $150 \mathrm{~km} \mathrm{~s}^{-1}$ shock propagating into an ambient medium of $n_{0}=100 \mathrm{~cm}^{-3}$ and $B_{0}=10 \mu \mathrm{G}$. [Fe II] $1.644 \mu \mathrm{m}$ and $\mathrm{H} \beta$ line emissivities are overplotted in an arbitrary linear scale. (Bottom left) Profiles of $\mathrm{H}$ nuclei density $(n)$, electron density $\left(n_{e}\right)$ and fraction of $\mathrm{Fe}$ in $\mathrm{Fe}^{+}\left(\mathrm{Fe}^{+} / \mathrm{Fe}\right)$ for the same shock. (Right) Synthesized IR spectrum of [Fe II] lines from the shock, normalized to the [Fe II] $1.644 \mu \mathrm{m}$ line intensity. The calculation is done by using the Raymond code.

spaces are available in Hollenbach et al. (1989), Mouri et al. (2000), and Allen et al. (2008). A grid of shock models with updated atomic parameters is in preparation by the author of this paper.

\subsection{NIR [Fe II] Observations of SNRs}

The first detection of the [Fe II] $1.644 \mu \mathrm{m}$ line in an SNR was reported by Seward et al. (1983) on MSH 15-52. After that, about a dozen Galactic and LMC SNRs have been observed in NIR [Fe II] lines. This number will increase with the completion of the UWIFE (UKIRT Wide-field Infrared Survey for $\mathrm{Fe}^{+}$) project, which is an unbiased survey of the [Fe II] $1.644 \mu \mathrm{m}$ line of the inner Galactic plane $\left(\ell=7^{\circ}\right.$ to $\left.65^{\circ} ;|b| \leqslant 1.3^{\circ}\right)$ using the UKIRT 4-m telescope. The UWIFE project is a "cousin" of the UWISH2 project, which covers the same area in the $\mathrm{H}_{2} v=1 \rightarrow 0 \mathrm{~S}(1)$ line at $2.122 \mu \mathrm{m}$ (Froebrich et al. 2011). Lee, Y.-H. et al. (this volume) introduce the two projects and present preliminary results on SNRs. In short, there are 77 SNRs in this area, and about $20 \%-30 \%$ of them are detected in [Fe II] and/or $\mathrm{H}_{2}$ lines, more than half of which are new detections.

The SNRs bright in [Fe II] emission lines may be divided into two groups: (1) middleaged SNRs interacting with dense molecular (or atomic) clouds, and (2) young SNRs interacting with the dense circumstellar medium (CSM).

Middle-aged SNRs bright in [Fe II] emission. Prototypical SNRs belonging to this category are W44, 3C391, and IC 443. All of them are interacting with molecular clouds (MCs). An indication of the MC interaction in the NIR band is the presence of $\mathrm{H}_{2}$ rovibrational emission lines that arise from slow, non-dissociative $\mathrm{C}$ shocks propagating into dense molecular gas of low-fractional ionization. Therefore, these middle-aged SNRs that are bright in [Fe II] emission are also bright in $\mathrm{H}_{2}$ emission (Fig. 3).

Figure 3 shows [Fe II] $1.644 \mu \mathrm{m}$ and $\mathrm{H}_{2} 2.122 \mu \mathrm{m}$ line images of W44; we see that the overall morphologies of the SNR in the two lines are similar, although the former appears rather diffuse and confined to the SNR boundary, whereas the latter is considerably filamentary and fills the entire SNR (see also Reach et al. 2005). A detailed inspection shows that, in some areas, there is a good spatial correlation between the two, whereas in other areas, the correlation is less significant. The detection of both [Fe II] and $\mathrm{H}_{2}$ 

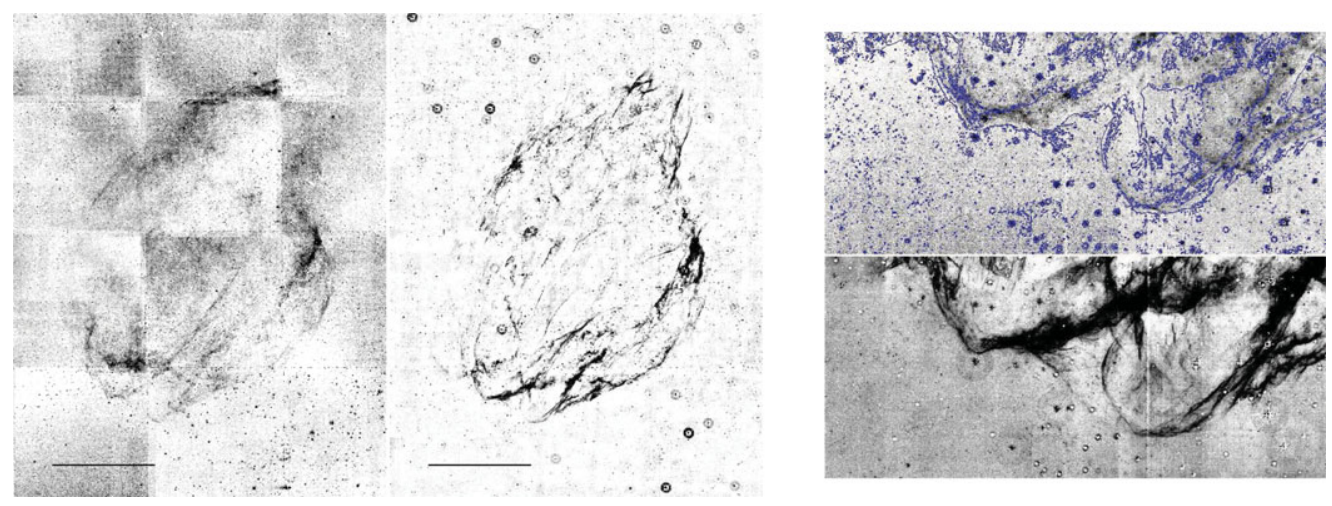

Figure 3. UWIFE [Fe II] $1.644 \mu \mathrm{m}$ (left) and UWISH2 $\mathrm{H}_{2} 2.122 \mu \mathrm{m}$ (middle) images of W44. The scale bar corresponds to $10^{\prime}$. The images on the right show zoomed-in views of the southern area in the [Fe II] (top) and $\mathrm{H}_{2}$ (bottom) emissions, respectively. The contours of the $\mathrm{H}_{2}$ emission are overlaid on the top right [Fe II] image.

emission lines is consistent with the general consensus that a molecular cloud is clumpy, being composed of dense clumps embedded in a rather diffuse interclump gas. An SNR produced inside a MC expands into the interclump medium while engulfing dense clumps. In late stages, the SNR shock in the interclump medium becomes radiative, so that the SNR becomes surrounded by a fast-expanding $\left(\sim 100 \mathrm{~km} \mathrm{~s}^{-1}\right)$ atomic shell, which is observable in Hi 21-cm line (e.g., Koo \& Heiles 1995; Chevalier 1999). The [Fe II] emission in such SNRs can originate in two regions; either from radiative atomic shocks in the interclump medium or from reflected shocks in the SNR shell generated by the interaction with molecular clumps. A detailed, comparative study of [Fe II] and $\mathrm{H}_{2}$ emissions should reveal more detailed information about the structure of molecular clouds.

Young SNRs bright in [Fe II] emission. There are also young SNRs bright in [Fe II] emission. Prototypical ones are Cas A, G11.2-0.3, RCW 103, and W49B, all of which are core-collapse SNRs (CCSNRs) with a central stellar remnant, except W49B, where no central source has been detected. One way to infer the environment of these [Fe II]bright young SNRs is to inspect diagrams such as Figure 4, where we compare the radii and ages of young SNRs. (For a discussion of SN types of young SNRs, see Chevalier 2005.) As in the figure, there is a trend of increasing SNR size with age, but with a large scatter, indicating diverse SN environments and also possibly diverse SN explosion energies. Among Type Ia SNRs, SN 1006 is located about 500 pc above the Galactic plane where the ambient density is $\sim 0.05 \mathrm{~cm}^{-3}$, whereas the Kepler SNR is interacting with a relatively dense $\left(\gtrsim 1 \mathrm{~cm}^{-3}\right)$ medium. Young CCSNRs are interacting either with CSM or a wind bubble created in the main-sequence lifetime of their progenitors. Cas A, which is Type IIb SN, for example, is interacting with a dense red supergiant (RSG) wind. The solid line is a model for Cas A from Chevalier \& Oishi (2003), but assuming $\dot{M}_{w}=3 \times 10^{-5} M_{\odot} \mathrm{yr}^{-1}, v_{w}=15 \mathrm{~km} \mathrm{~s}^{-1}, M_{\mathrm{ej}}=5 M_{\odot}$, and $E_{\mathrm{SN}}=10^{51}$ ergs where $\dot{M}_{w}$ is the wind mass-loss rate, $v_{w}$ is the wind speed, $M_{\mathrm{ej}}$ is the ejecta mass, and $E_{\mathrm{SN}}$ is the explosion energy. Cas A follows this "Cas A-like" line as long as it continues to interact with the dense CS wind. There are CCSNRs which fall well below the Cas A-like line, i.e., G11.2-0.3, RCW 103, and G292.0+1.8. Note that the first two SNRs are bright in [Fe II] lines. (G292.0+1.8 has not been observed in [Fe II] emission.) Their relatively small sizes could be due to either a small explosion energy $\left(<10^{50} \mathrm{ergs}\right)$ or/and dense CSM. The strong [Fe II] lines in these SNRs suggest that it is more likely because of dense CSM and that the CSM is much denser than that of Cas A. Hence, they are likely the 

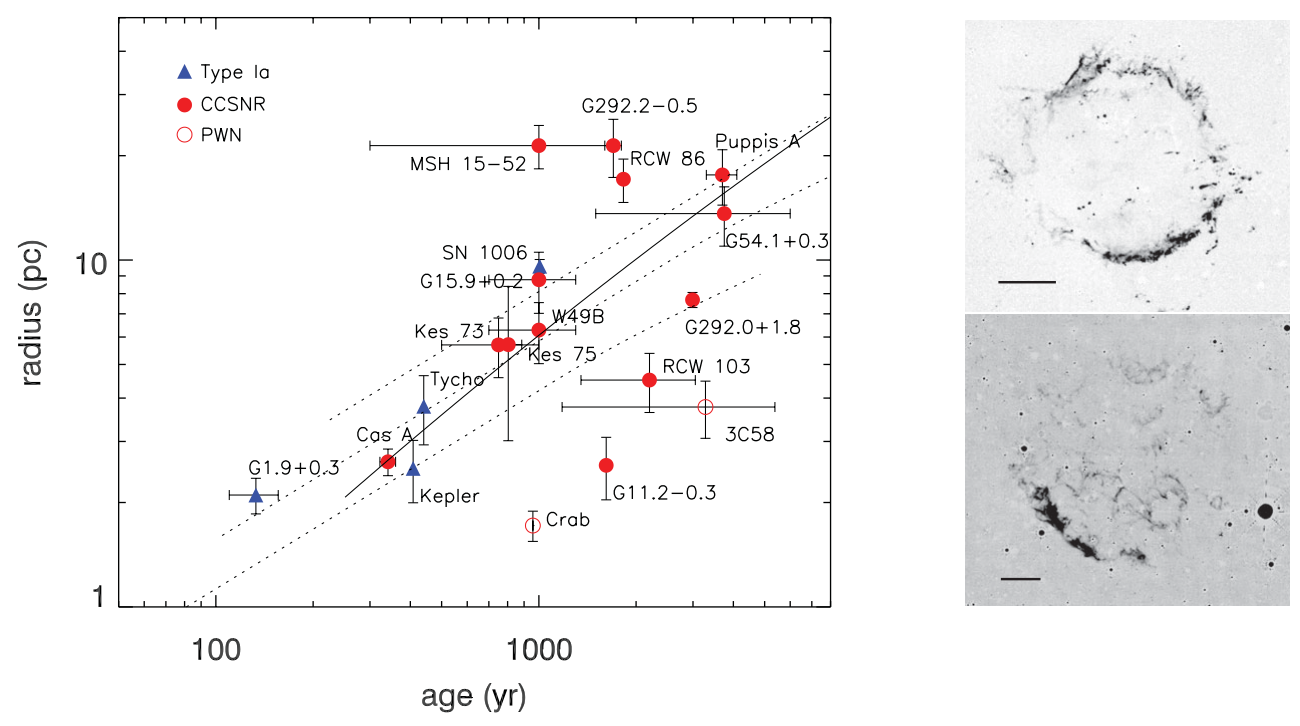

Figure 4. (Left) Radius versus age of young SNRs. The dotted lines are models for SNRs in uniform ambient media of $n_{0}=1,0.1$, and $0.01 \mathrm{~cm}^{-3}$, respectively (Truelove \& McKee 1999; $n=7$ ejecta model with $M_{\mathrm{ej}}=5 M_{\odot}$ and $\left.E_{\mathrm{SN}}=10^{51} \mathrm{ergs}\right)$. The solid line is for an SNR in the RSG wind case (Chevalier\& Oishi 2003; see text for the parameters of the model.) Note that the ones marked by empty circles are pulsar wind nebulae, so they do not represent true sizes of SNRs. (Right) [Fe II] images of Cas A and G11.2-0.3 from top to bottom. The scale bars correspond to $1 \mathrm{pc}$ at the distances of the SNRs.

remnants of massive SN IIL/b or SN IIn. On the other hand, there are remnants much larger than Cas A: MSH 15-52, G292.2-0.5, and RCW 86. These remnants might have exploded inside a large bubble, and thus, they are candidates for $\mathrm{SN} \mathrm{Ib/c,} \mathrm{although} \mathrm{a}$ large SN explosion energy (>10 $10^{52} \mathrm{ergs}$ ) could be another possibility. Figure 4 suggests that all four [Fe II]-bright young CCSNRs mentioned at the beginning of this paragraph are SN IIL/b candidates interacting with dense CSM. (For W49B, however, the bipolar Type Ib/c SN origin has been suggested. See Lopez et al. 2013 and references therein.)

The [Fe II] emission in these young SNRs originate from both shocked CSM and shocked SN ejecta. In Cas A, it is well known that there are two types of knots detected in the visible waveband: quasi-stationary flocculi (QSFs), which are dense CS knots moving at a few hundred kilometers per second, and fast moving knots (FMKs), which are metal-rich SN ejecta knots moving at several thousand kilometers per second. Lee, Y.-H. et al. (this volume) show that there are also fast-moving [Fe II] knots that lack other metallic lines, which could be pure Fe ejecta synthesized in the innermost SN region. In G11.2-0.3, which is known as a cousin of Cas A because of its similar morphology (Fig. 4), the knots in the central area have radial velocities of $\sim 1,000 \mathrm{~km} \mathrm{~s}^{-1}$, which suggests that they might be SN ejecta (Moon et al. 2009). Again, their spectra do not show metallic lines other than Fe. The bright filament in the southeast of G11.2-0.3, on the other hand, appears to be composed of mostly dense CSM. For the [Fe II] emission features in RCW 103 and W49B, detailed spectroscopic studies are yet to be performed (cf. Oliva et al. 1999; Keohane et al. 2007).

\subsection{Some Issues}

[Fe II] $-\mathrm{H}_{2}$ reversal . Since the early days of NIR observations of SNRs, it has been known that there are SNRs with $\mathrm{H}_{2}$ filaments lying beyond [Fe II] filaments, i.e., further out from the SNR center, which is not easily explained by shock models (Graham et al. 
1991; Oliva, Moorwood, \& Danziger 1990; Burton \& Spyromilio 1993). We now have more sources showing similar patterns, e.g., G11.2-0.3, W49B, and 3C396 (Koo et al. 2007; Keohane et al. 2007; Lee et al. 2009). W44 in Figure 3 is another example. Hence, we need an explanation for this "[Fe II] $-\mathrm{H}_{2}$ reversal"; i.e., we need to know what the exciting mechanisms of the $\mathrm{H}_{2}$ emission is and how they excite the $\mathrm{H}_{2}$ gas beyond the SNR. Some proposed mechanisms are fluorescent UV excitation, X-ray heating, magnetic precursors, and reflected shocks, but high-resolution NIR spectroscopic studies are needed to address the issue.

[Fe II] luminosity as an SN rate indicator. Can the [Fe II] 1.257 or $1.644 \mu \mathrm{m}$ luminosities be used as an indicator of galactic SN rates? Several groups have addressed this for starburst galaxies and have derived a conversion factor of SN rate $=0.03-0.1$ $L_{[F e I I]} / 10^{6} L_{\odot} \mathrm{yr}^{-1}$ where $L_{[F e I I]}$ is the [Fe II] $1.644 \mu \mathrm{m}$ luminosity (Morel et al. 2002; Alonso-Herrero et al.2003; Rosenberg et al. 2012). In the case of nearby starburst galaxies M82 and NGC 253, however, $70 \%-80 \%$ of the [Fe II] emission is known to be diffuse emission of unknown origin, although it is speculated to be related to the SN activity therein (Greenhouse et al. 1997; Alonso-Herrero et al. 2003). In the Galaxy, the [Fe II]bright SNRs represent 20\%-30\% of the known $~ 300$ SNRs, which occupy a small fraction of the entire set of SNRs present in the Galaxy. It is clear that we need to have better understanding of the population of [Fe II]-bright SNRs and also the origin of the diffuse [Fe II] emission to obtain a more reliable relation between $L_{[F e I I]}$ and the $\mathrm{SN}$ rate in galaxies.

\section{Dust IR Emission from SNRs}

\subsection{Dust Heating in SNRs Interacting with MCs}

Dust in SNRs can be heated either collisionally or radiatively. In SNRs with fast, nonradiative shocks, dust grains are heated by collisions with gas particles, mainly electrons, in a hot plasma behind the shocks (e.g., Dwek et al. 2008). Many SNRs have mid- and far-IR morphology almost identical to that of X-ray, which suggests that the IR emission in these SNRs is primarily from collisionally-heated dust grains.

SNRs interacting with MCs generally have IR morphology different from the X-ray morphology; they appear shell-like in IR whereas they are centrally brightened in X-rays (Fig. 5). The dust in these SNRs cannot be collisionally-heated by X-ray emitting gas. Instead the likely source of the heating is radiation from the shock front. In radiative
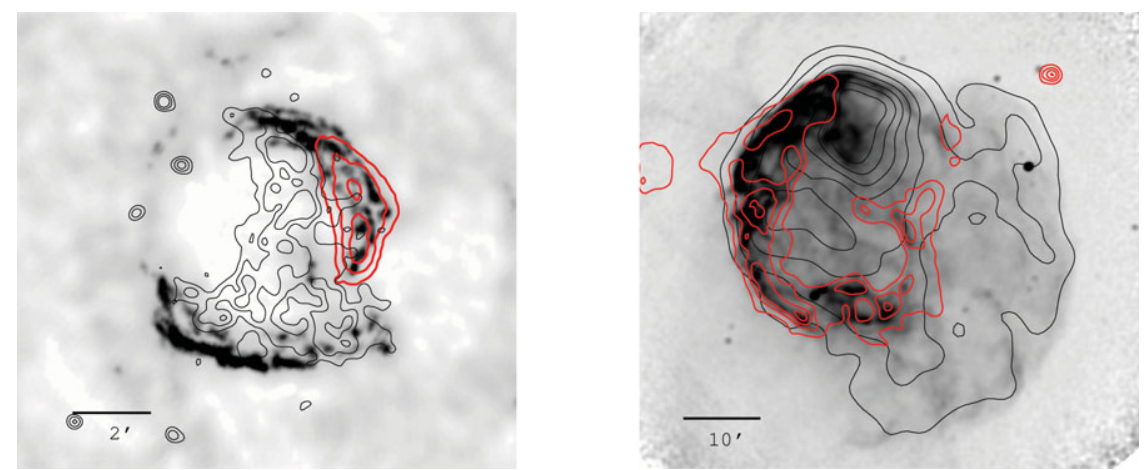

Figure 5. (Left) ATCA 20-cm image of Kes 17 with overlaid XMM 0.2-12 keV X-ray (thin) and AKARI $65 \mu \mathrm{m}$ (thick) contours. (See also Lee et al. 2011.) (Right) VLA 20-cm image of IC 443 with overlaid ROSAT 0.2-2.4 keV X-ray (thin) and AKARI $90 \mu \mathrm{m}$ (thick) contours. 
shocks, the UV radiation from the cooling postshock gas could be much stronger than the general interstellar radiation field (e.g., McKee et al. 1987). This UV radiation, dominated by trapped Ly $\alpha$ photons, heat the dust in the cooling layer, and, subsequently, the infrared radiation from these hot dust grains heats the dust at larger column densities (e.g., Hollenbach et al. 1979). The far-IR bright regions in MC-interacting SNRs (Fig. 5) are probably where the radiation field is strong and the ambient density is high. Andersen et al. (2011) carried out a systematic study of the dust emission from MC-interacting SNRs found in the GLIMPSE survey, and derived dust temperatures of 29-66 K from the Spitzer MIPS spectral energy distribution $(60-90 \mu \mathrm{m})$. There could be a dust component at a lower temperature, however, because the SNRs interacting with MCs are bright in the far-IR waveband beyond the MIPS coverage (e.g., see Lee et al. 2011). A systematic study of MC-interacting SNRs, including the AKARI and Herschel far-IR data, will be useful to understand the heating mechanisms and also the processing of dust grains in these SNRs.

\subsection{Star Dust in SNRs}

SN dust in young CCSNRs. The dense, metal-rich, cooling SN ejecta can effectively provide an environment for dust to condense. In the high-redshift galaxies, where low-mass stars do not have enough time to evolve to AGB stars, SNe could be the main contributors of dust, depending on the dust yield (Dwek \& Cherchneff 2011). Theoretical studies have shown that as much as $1 M_{\odot}$ of different dust species can form in SN IIP with massive $\mathrm{H}$ envelopes, whereas in SN IIL/b or SN Ia with little or no $\mathrm{H}$ envelopes only a limited amount of dust could form (Nozawa et al. 2010; Nozawa et al. 2011).

In observational studies, however, only a very small amount of dust in SNe has been detected, i.e., $\lesssim 10^{-3} M_{\odot}$. (see Gall et al. 2011 and references therein). It is only toward the LMC SN 1987A and some young Galactic SNRs where a significant amount of SN dust has been detected: In 1987A, Matsuura et al. (2011) reported detection of $0.4-$ $0.7 M_{\odot}$ of dust, whereas, in the Galaxy, $0.1-0.2 M_{\odot}$ of dust has been detected in Cas A and the Crab nebula which are SNIIb and SN IIP(?), respectively. In another SN IIP candidate, G54.1+0.3, a dust ring of $0.58-0.86 M_{\odot}$ has been detected around its pulsar wind nebula, but the nature of the ring is not yet conclusively identified (Koo 2012).

Crystalline silicate dust in MSH 15-52. Essentially all dust grains in the ISM are amorphous. Crystalline silicate dust grains have been found mainly in evolved stars and young stellar objects, indicating that they form in situ in circumstellar disks and/or outflows of these objects (Henning 2010). In this regard, the detection of crystalline silicates in the SNR MSH 15-52 is interesting (Koo et al. 2011).

As we mentioned in $\S 2.2$, MSH $15-52$, is a young ( $1000 \mathrm{yr})$ SNR probably expanding inside a bubble, suggesting progenitor SN type of Ib/c (see Fig. 4). The remnant has a central pulsar, and there is an O star (Muzzio 10) and a bright MIR source (IRAS 15099-5856) lying very close from the pulsar, i.e., $18^{\prime \prime} \& 31^{\prime \prime}$ (or 0.35 pc \& 0.60 pc at $4 \mathrm{kpc}$ ) to north, respectively. IRAS $15099-5856$ is probably a dusty cloud heated by Muzzio 10. What is special to this mid-IR source is that it has prominent crystalline silicate spectral features. Koo et al. (2011) proposed a scenario where the SN progenitor and Muzzio 10 were in a binary system and IRAS 15099-5856 is a CSM survived from the SN blast wave due to the shielding by Muzzio 10 (see Koo 2012). MSH 15-52 appears to be the first case in which crystalline silicates associated with a SNR have been observed.

Acknowledgments I wish to thank Lee, Y.-H., Jeong, I.-G, and Moon, D.-S. for their help with figures. My research is supported by Basic Science Research Program through 
the National Research Foundation of Korea (NRF) funded by the Ministry of Education, Science and Technology (NRF-2011-0007223).

\section{References}

Allen, M. G., Groves, B. A., Dept, M. A., Sutherland, R. S., \& Kewley, L. J. 2008, ApJS, 178, 20

Alonso-Herrero, A., Rieke, G. H., Rieke, M. J., \& Kelly, D. M. 2003, ApJ, 125, 1210

Andersen, M., Rho, J., Reach, W. T., Hewitt, J. W., \& Bernard, J. P. 2011, ApJ, 742, 7

Burton, M. \& Spyromilio, J. 1993, Proceedings of the Astronomical Society of Australia, 10, 327

Chevalier, R. A. 1999, ApJ, 511, 798

Chevalier, R. A. 2005, ApJ, 619, 839

Chevalier, R. A. \& Oishi, J. 2003, ApJL, 593, L23

Dwek, E., Arendt, R. G., Bouchet, P., et al. 2008, ApJ, 676, 1029

Dwek, E. \& Cherchneff, I. 2011, ApJ, 727, 63

Froebrich, D., Davis, C. J., Ioannidis, G., et al. 2011, MNRAS, 413, 480

Gall, C., Hjorth, J., \& Andersen, A. C. 2011, A\& ARv, 19, 43

Graham, J. R., Wright, G. S., Hester, J. J., \& Longmore, A. J. 1991, AJ, 101, 175

Greenhouse, M. A., Satyapal, S., Woodward, C. E., et al. 1997, ApJ, 476, 105

Henning, T. 2010, ARAA, 48, 21

Hollenbach, D. J., Chernoff, D. F., \& McKee, C. F. 1989, Infrared Spectroscopy in Astronomy, 290,245

Hollenbach, D. \& McKee, C. F. 1979, ApJS, 41, 555

Keohane, J. W., Reach, W. T., Rho, J., \& Jarrett, T. H. 2007, ApJ, 654, 938

Koo, B.-C. 2012, Publication of Korean Astronomical Society, 27, 225

Koo, B.-C. \& Heiles, C. 1995, ApJ, 442, 679

Koo, B.-C., McKee, C. F., Suh, K.-W., et al. 2011, ApJ, 732, 6

Koo, B.-C., Moon, D.-S., Lee, H.-G., Lee, J.-J., \& Matthews, K. 2007, ApJ, 657, 308

Lee, H.-G., Moon, D.-S., Koo, B.-C., et al. 2011, ApJ, 740, 31

Lee, H.-G., Moon, D.-S., Koo, B.-C., Lee, J.-J., \& Matthews, K. 2009, ApJ, 691, 1042

Lopez, L. A., Ramirez-Ruiz, E., Castro, D., \& Pearson, S. 2013, ApJ, 764, 50

Matsuura, M., Dwek, E., Meixner, M., et al. 2011, Science, 333, 1258

McKee, C. F., Hollenbach, D. J., Seab, G. C., \& Tielens, A. G. G. M. 1987, ApJ, 318, 674

Moon, D.-S., Koo, B.-C., Lee, H.-G., et al. 2009, ApJ, 703, L81

Morel, T., Doyon, R., \& St-Louis, N. 2002, MNRAS, 329, 398

Mouri, H., Kawara, K., \& Taniguchi, Y. 2000, ApJ, 528, 186

Nozawa, T., Kozasa, T., Tominaga, N., et al. 2010, ApJ, 713, 356

Nozawa, T., Maeda, K., Kozasa, T., et al. 2011, ApJ, 736, 45

Oliva, E., Moorwood, A. F. M., \& Danziger, I. J. 1989, A\& $A, 214,307$

Oliva, E., Moorwood, A. F. M., \& Danziger, I. J. 1990, A\& A, 240, 453

Oliva, E., Moorwood, A. F. M., Drapatz, S., Lutz, D., \& Sturm, E. 1999, A\&\&A, 343, 943

Pradhan, A. K. \& Nahar, S. N. 2011, Atomic Astrophysics and Spectroscopy Cambridge University Press: Cambridge and New York

Reach, W. T., Rho, J., \& Jarrett, T. H. 2005, ApJ, 618, 297

Rosenberg, M. J. F., van der Werf, P. P., \& Israel, F. P. 2012, AESA, 540, A116

Seward, F. D., Harnden, F. R., Jr., Murdin, P., \& Clark, D. H. 1983, ApJ, 267, 698

Truelove, J. K. \& McKee, C. F. 1999, ApJS, 120, 299

\section{Discussion}

PODSIADLOWSKI: What is the estimated mass of the O-star companion in MSH15-52?

Koo: It's spectral type is O4.5 III and the mass is 45 MSun 